Results: To detect the most effective JAKi in blocking the inflammatory response induced by IL1- $\beta$, RA-FLS were first pretreated with different JAKi for 2 hour with concentrations of $1 \mu \mathrm{M}$ and $10 \mu \mathrm{M}$ and then additionally stimulated with IL1- $\beta$ $(20 \mathrm{ng} / \mathrm{ml}$ ) for 18 hour. Even at the highest concentration of $10 \mu \mathrm{M}$ Tofacitinib and Baricitinib did not change the IL- 6 levels, whereas Peficitinib and Filgotinib reduced the IL-6 release at $10 \mu \mathrm{M}$. Tofacitinib and Baricitinib reduced the cytokine release if the RA-FLS were stimulated with OSM, a factor directly inducing the JAK-dependent IL-6-pathway $(n=3)$.

To obtain a dose-response curve for the clinically relevant range of concentrations between $0.01 \mu \mathrm{M}$ and $5 \mu \mathrm{M}$, RA-FLS were pretreated with Filgotinib and Peficitinib for 2 hour and then stimulated with IL $1-\beta(10 \mathrm{ng} / \mathrm{ml})$ for 17 hour. In contrast to Filgotinib, Peficitinib at $5 \mu \mathrm{M}$ caused a reduction of IL- 6 levels of $66 \%$ compared to control with IL1- $\beta(p<0.01, n=5)$. The MMP-3 release was decreased by both substances at $5 \mu \mathrm{M}$ : In comparison to the control with IL1- $\beta$, Peficitinib caused a reduction of $92 \%(p<0.0001, n=5)$ whereas Filgotinib only reduced the levels by $43 \%(p<0.05, n=3)$. Furthermore, Peficitinib at $1 \mu \mathrm{M}$ decreased the MMP-3 release by $46 \%(p<0.01)$.

The treatment with Peficitinib did not affect the viability, cytotoxicity or apoptosis of RA-FLS $(n=3)$. Therefore, the effects of Peficitinib on the inflammatory response were not caused by cell death.

Conclusions: Peficitinib reduced the release of proinflammatory cytokines and of matrix metalloproteinases after activation of RA-FLS with IL1- $\beta$ and appeared to be superior to Tofacitinib and Baricitinib in targeting the pro-inflammatory and matrix destructive properties of RA-FLS.

Disclosure of Interest: None declared

DOI: 10.1136/annrheumdis-2018-eular.2182

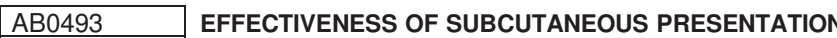 OF METHOTREXATE IN PATIENTS WITH RHEUMATOID ARTRHITIS}

L. Villarreal ${ }^{1}$, M. Rivero $^{2}$, D. Buitrago-Garcia ${ }^{3}$, M. Cabrera ${ }^{1}$, P. Santos-Moreno ${ }^{4}$. ${ }^{1}$ Health Services; ${ }^{2}$ Pharmaceutical Chemist, ${ }^{3}$ Epidemiology; ${ }^{4}$ Rheumatology, Biomab, Center For Rheumatoid Arthritis, Bogota, Bogota, Colombia

Background: Methotrexate (MTX) is one of the most used drugs for the treatment of rheumatoid arthritis (RA) and has become the gold standard of therapy. It can be given via oral, intramuscular or subcutaneous (SC). MTX is a highly effective therapy for patients with RA; however, oral MTX has been associated with gastrointestinal intolerance diminishing the adherence of patients to treatment and increasing the switching to biological therapy. Thus, the use of SC methotrexate can improve its efficacy compared to oral MTX.

Objectives: The aim was to determine the effectiveness and safety of SC MTX in patients with rheumatoid arthritis.

Methods: We performed a retrospective descriptive analysis; our main goal was to provide real-life data regarding effectiveness of SC MTX in patients with RA. We excluded patients who were in remission. Clinical follow-up was designed by the authors according to DAS28 as follows: every $3-5$ weeks (DAS28 $>5.1$ ), every $7-9$ weeks (DAS28 $\geq 3.1$ and $\leq 5.1$ ), and every $11-13$ weeks (DAS28 $<3.1$ ). Tender joint count (TJC), swollen joint count (SJC) and DAS28 were measured on each visit. Therapy had to be adjusted with DAS28 $>3.2$ unless patient's conditions don't permit it; regarding the effectiveness of SC MTX we calculated means, and standard deviations for continuous variables and categorical variables were presented as rates. We performed a bivariate analysis using Pearson's chi ${ }^{2}$

Abstract AB0493 - Table 1. DAS28 and CDAl in patients with RA receiving SC MTX

\begin{tabular}{lcccc}
\hline & \multicolumn{2}{c}{ BASELINE } & \multicolumn{2}{c}{ 12 MONTHS } \\
\hline DAS 28 & $\mathrm{n}$ & $\%$ & $\mathrm{n}$ & $\%$ \\
\hline Remission & & & 44 & 33 \\
LDA & 36 & 28 & 29 & 22 \\
MDA & 84 & 63 & 50 & 38 \\
SDA & 12 & $\%$ & 9 & 7 \\
CDAl & $\mathrm{n}$ & & $\mathrm{n}$ & $\%$ \\
Remission & & 66 & 38 & 29 \\
Low & 87 & 26 & 62 & 47 \\
Moderate & 34 & 8 & 25 & 19 \\
High & 11 & & 7 & 5 \\
\hline
\end{tabular}

Results: A total of 132 patients meet our inclusion criteria, $88 \%$ were female and $12 \%$ male. Mean age was 61 years \pm 9 . Mean DAS28 at baseline was $3.8 \pm 0.83$, while at 12 months mean DAS28 was $3.28 \pm 1.12$. Clinical disease activity CDAl at baseline had a mean of $9.6 \pm 7.2$ and at 12 months $7.8 \pm 7.5$. We achieved remission in $33 \%$ of patients according to DAS28\% and $29 \%$ according to CDAI, also it was a significant decrease in patients with moderate disease activity see table 1. Finally, there was a statistical significance between disease activity at baseline compared to disease activity at 12 months $(p<0.005)$. It was not observed major complaints with SC MTX, only $5 \%$ of the patients reported some mild and transient discomfort at the local application site.

Conclusions: Subcutaneous MXT is an effective and safe alternative for the treatment in patients with RA and intolerance to oral MTX, and could be a good option to prevent a premature switching to biological therapy.

Disclosure of Interest: None declared

DOI: 10.1136/annrheumdis-2018-eular.7300

\section{AB0494 USABILITY OF A PRE-FILLED PEN FOR SELF- ADMINISTRATION OF SUBCUTANEOUS METHOTREXATE}

\section{Hattesohl, M. Tribanek, K. Gescher. MEDAC GMBH, Wedel, Germany}

Background: Methotrexate (MTX) is the first-line DMARD for the treatment of rheumatoid arthritis (RA). ${ }^{1}$ It is also of high significance in the treatment of psoriasis arthritis (PsA) and psoriasis vulgaris (PsV). ${ }^{23}$

Studies in RA patients found that there are advantages in terms of bioavailability and efficacy when MTX is administered subcutaneously (SC) as compared to orally. ${ }^{4,5}$

Since the introduction of MTX pre-filled syringes, patients are principally enabled to self-administer methotrexate subcutaneously, however not all patients are able to use syringes easily. Auto-injection using a pre-filled pen can facilitate administration by the patient. Results of a comparative crossover study showed that SC self-injection with a pre-filled pen is mainly preferred to a pre-filled syringe with regard to use, acceptability, and satisfaction ${ }^{6}$. Usability of the pre-filled pen was proven in an actual-use study ${ }^{7}$, however no comprehensive data from routine clinical use is available so far.

Objectives: The aim of this practice documentation was the evaluation of the usability of a MTX pre-filled pen under everyday clinical conditions.

Methods: This multicentric non-interventional practice documentation, which took place from 2013 to 2015 in Germany, was open to naïve patients and to those who had already been treated with the pre-filled pen in accordance with the approved indications. Usability was evaluated by the patient and the physician through assessment of the individual steps of two self-injections in an interval of approximately 12 weeks (V1, V2) and through a final overall assessment using ordinal scales. Data analysis was carried out descriptively by means of standard statistical methods. Calculation of a total usability score allowed for comparison of V1 and V2.

Results: The 478 participating patients had a mean age of $56.2 \pm 13.7$ years, $57.1 \%$ were female; $39.3 \%$ suffered from RA, $34.7 \%$ from PsV, $23.4 \%$ had PsA, and $12.6 \%$ were diagnosed with other conditions. $87.7 \%$ received treatment with the pre-filled pen for the first time, and $61.5 \%$ had previously received MTX. The individual steps of self-injection, removing of a protective cap, placing the device onto the skin, pressing the injection button, removing the pen, and ergonomics of the pen, were rated as "very good" and "good" by more than $90 \%$ of patients as well as of physicians in both visits. Comparing V1 and V2, usability was assessed to be even better at the second visit $(p<0.0001$ in the total score). Injections were carried out accurately by more than $93 \%$ of the patients.

Conclusions: Autoinjection with a pre-filled pen enables patients to self-adminis ter subcutaneous MTX easily and comfortably in routine clinical practice.

\section{REFERENCES:}

[1] Krüger K, et al. Z Rheumatol 2012;71(7):592-603.

[2] Gossec L, et al. Ann Rheum Dis. 2016;75(3):499-510.

[3] Nast A, et al. J Eur Acad Dermatol Venereol 2015;29:2277-94.

[4] Schiff MH, et al. Ann Rheum Dis. 2014;73(8):1549-51.

[5] Braun J, et al. Arthritis Rheum. 2008;58(1):73-81.

[6] Demary W, et al. Patient Prefer Adherence 2014;8:1061-71.

[7] Pachon JA, et al. SAGE Open Med. 2014;2:2050312114564241.

Disclosure of Interest: M. Hattesohl Employee of: medac $\mathrm{GmbH}$, M. Tribanek Employee of: medac GmbH, K. Gescher Employee of: medac GmbH DOI: 10.1136/annrheumdis-2018-eular.6548 